\title{
Kinetic Simulation of Relativistic Radiative Steady-State Magnetic Reconnection
}

\section{José Ortuño-Macías \& Krzysztof Nalewajko}

Nicolaus Copernicus Astronomical Center, Polish Academy of Sciences, Bartycka 18, 00716

Warsaw, Poland

E-mail: jortuno@camk.edu.pl

\begin{abstract}
High energy astrophysical phenomena, such as blazars, gamma-ray burst and pulsar wind nebulae, show emission signatures of non-thermal particle distribution indicating efficient particle acceleration. They also share a common feature — strong magnetic fields with magnetic energy content of the order of the rest mass energy. Under such conditions relativistic magnetic reconnection (RMR) is the most efficient mechanism for particle acceleration. By means of kinetic simulations, previous studies of steady-state RMR have demonstrated its ability for producing highly energetic non-thermal particle distributions. These energetic particles are mainly contained within plasmoids, also known as magnetic islands. A chain of plasmoids with stochastic properties is continuously generated by tearing instability within the reconnecting layer. Plasmoid statistics have been used to model the broad band and highly variable emission observed in blazars. However in the regime of high emission efficiency, radiative cooling influences particle dynamics, and thus it may also affect plasmoids properties. We present the results of 2D PIC simulations of steady state RMR which include synchrotron radiation reaction and calculation of the resulting emission signatures. We show that radiative cooling affects mainly the dense cores of large plasmoids, which are also the main sites of synchrotron emission. Synchrotron lightcurves show rapid bright flares that can be identified as originating from tail-on mergers between a small/fast plasmoid and a large/slow target.
\end{abstract}

High Energy Phenomena in Relativistic Outflows VII - HEPRO VII

9-12 July 2019

Facultat de Física, Universitat de Barcelona, Spain 


\section{Introduction}

Blazars are classified as particular type of active galactic nuclei (AGN) in which the relativistic jet is pointing toward the observer. Their spectral emission is characterized by two non-thermal broad components, a low-energy one that goes from radio to UV/X-rays and a high-energy one that expands from X-rays to $\gamma$-rays, commonly modeled as inverse Compton scattering of various soft radiation fields. The emission also presents variability on timescales ranging from decades (Ahnen et al., 2016) to minutes (Aharonian et al., 2007), the latter being shorter than the light crossing timescale ( $\sim$ hours) of the supermassive black hole (SMBH) located at the base of the jet. In the case of $\mathrm{TeV}$ emission, in order for such photons to escape from the jet without producing positron-electron pairs due to photon-photon absorption in the soft radiation fields, it is deduced that the bulk Lorentz factor of the emitting flow must be $\gtrsim 50$ (Begelman et al., 2008). However, the observed AGN jets at sub-pc scales commonly have Lorentz factors of 10-20, so an additional relativistically outflowing region within the co-moving jet flow would be required. These suggest that the blazar $\mathrm{TeV}$ emission is coming from compact highly magnetized regions within the jet, where particles are being efficiently accelerated.

The RMR has been proposed as the magnetic energy dissipation mechanism that can operate in AGN jets and produce localized relativistic outflows called 'minijets' (Giannios et al., 2009; Nalewajko et al., 2011). High particle acceleration efficiency by means of RMR has been demonstrated using kinetic particle-in-cell (PIC) simulations (e.g., Sironi, \& Spitkovsky, 2014). Usually these simulations have been carried out with periodic boundary conditions, which eventually influence the reconnection rate and limit the reconnection outflows and generation of plasmoids. More recently, PIC simulations have been carried out with open boundaries, achieving a steady-state RMR with unimpeded outflows (Sironi et al., 2016). It has been shown that a chain of plasmoids with stochastic properties is generated. These stochastic properties, such as size, momentum and energy content, allows for a realistic description of the multi-wavelength and multi-timescale blazar emission, as shown with the post-analysis emission models (Petropoulou et al., 2016; Christie et al., 2019).

However, PIC simulations which include radiative effects have proved that under high radiative efficiency the capability for particle acceleration is reduced, as compared with the previous nonradiative simulations (Nalewajko, 2018; Schoeffler et al., 2019; Hakobyan et al., 2019). Hence, the plasmoids properties may also be affected by strong radiative cooling. Here, we present preliminary results of PIC simulations of the steady-state RMR, including radiation reaction and emission signatures produced self-consistently by the synchrotron process. 


\section{Simulation setup}

We make use of a custom version of the PIC code Zeltron (Cerutti et al., 2013) to perform 2D kinetic simulations of steady-state RMR in pair plasma, electron and positron plasma. The initial stage involves a single Harris-type current layer placed in the middle of the computational domain (Kirk, \& Skjæraasen, 2003)

$$
\begin{array}{r}
B_{\text {ini,x }}=-B_{0} \tanh (y / \delta) \\
n_{\mathrm{d}}(y)=n_{\mathrm{d}, 0} \cosh ^{-2}(y / \delta)
\end{array}
$$

In order to trigger the RMR process at the middle of the current layer, a small current and pressure gap is set driving that region to dynamical collapse. The steady state is achieved by open boundaries, where the particles are able to escape, the fields are absorbed, and particles are injected at rate that matches the initial density and current profiles. At every time step we perform the following operation:

$$
\begin{aligned}
& \mathbf{B}(x) \rightarrow \mathbf{B}(x)+\lambda(x)\left[\mathbf{B}_{i n i}(x)-\mathbf{B}(x)\right] \\
& \mathbf{E}(x) \rightarrow \mathbf{E}(x)+\lambda(x)\left[\mathbf{E}_{i n i}(x)-\mathbf{E}(x)\right]
\end{aligned}
$$

where $\lambda(x)=0.5\left(\left|x-x_{a b s}\right| / \Delta_{a b s}\right)^{3}, x_{a b s}$ is the position of the absorbing layer inner edge and $\Delta_{a b s}$ is the field-absorbing layers thickness.

The synchrotron radiation signatures are computed at every time step, accounting for the radiation reaction force that emitting particles experience. Simultaneously, the spectral synchrotron emission from the particle ensemble is collected as it would be measured by two observers placed at the edges of the current layer where the relativistic outflowing plasma emission is beamed.

We explore high cooling efficiency by means of different plasma configurations of the particle temperature $\Theta=k T / m c^{2}$, where the nominal cooling length is of the order or smaller than the computational domain size, $l_{\text {cool }}<L_{x}$. Synchrotron cooling length is defined similar to Nalewajko (2018) with the initial background magnetic energy density $U_{\mathrm{B}, 0}=\left\langle B^{2}\right\rangle /(8 \pi)=B_{0}^{2} /(8 \pi)$,

$$
l_{\mathrm{cool}}=c \tau_{\mathrm{cool}}=\frac{\langle\gamma\rangle}{\langle|\mathrm{d} \gamma / c \mathrm{~d} t|\rangle} \simeq \frac{\langle\gamma\rangle}{\left\langle\gamma^{2}\right\rangle} \frac{3 m_{e} c^{2}}{4 \sigma_{T} U_{\mathrm{B}, 0}} \simeq \frac{(3 \pi / 2) e}{\sigma_{T} \Theta^{2} B_{0}} \rho_{0}
$$

The results presented correspond to a simulation with plasma initial temperature set to $\Theta=5$. $10^{5}$, magnetization parameter defined as $\sigma=B_{0}^{2} / 4 \pi m n_{0} c^{2} \Theta$ set to $\sigma=10$, magnetic field $B_{0}=1 G$. The size of the computational domain in physical units is $L_{x} / \rho_{0}=1536$ where $\rho_{0}$ is the plasma nominal gyroradius given by $\rho_{0}=\Theta m_{e} c^{2} /\left(e B_{0}\right)$. The corresponding cooling length to domain size ratio is $l_{\text {cool }} / L_{x} \simeq 0.3$.

\section{Results}

In top panel of Fig. 1 we show number density snapshots and magnetic field lines of the initial evolution for the reconnecting layer. Each panel presents the central region of our computational 
domain where the current layer is placed. It can be appreciated that the triggering starts reconnection in the middle of the current layer while expanding a central region of low-density sustained by a thin current layer that is unstable to tearing modes leading to a continuous chain of plasmoids generation.
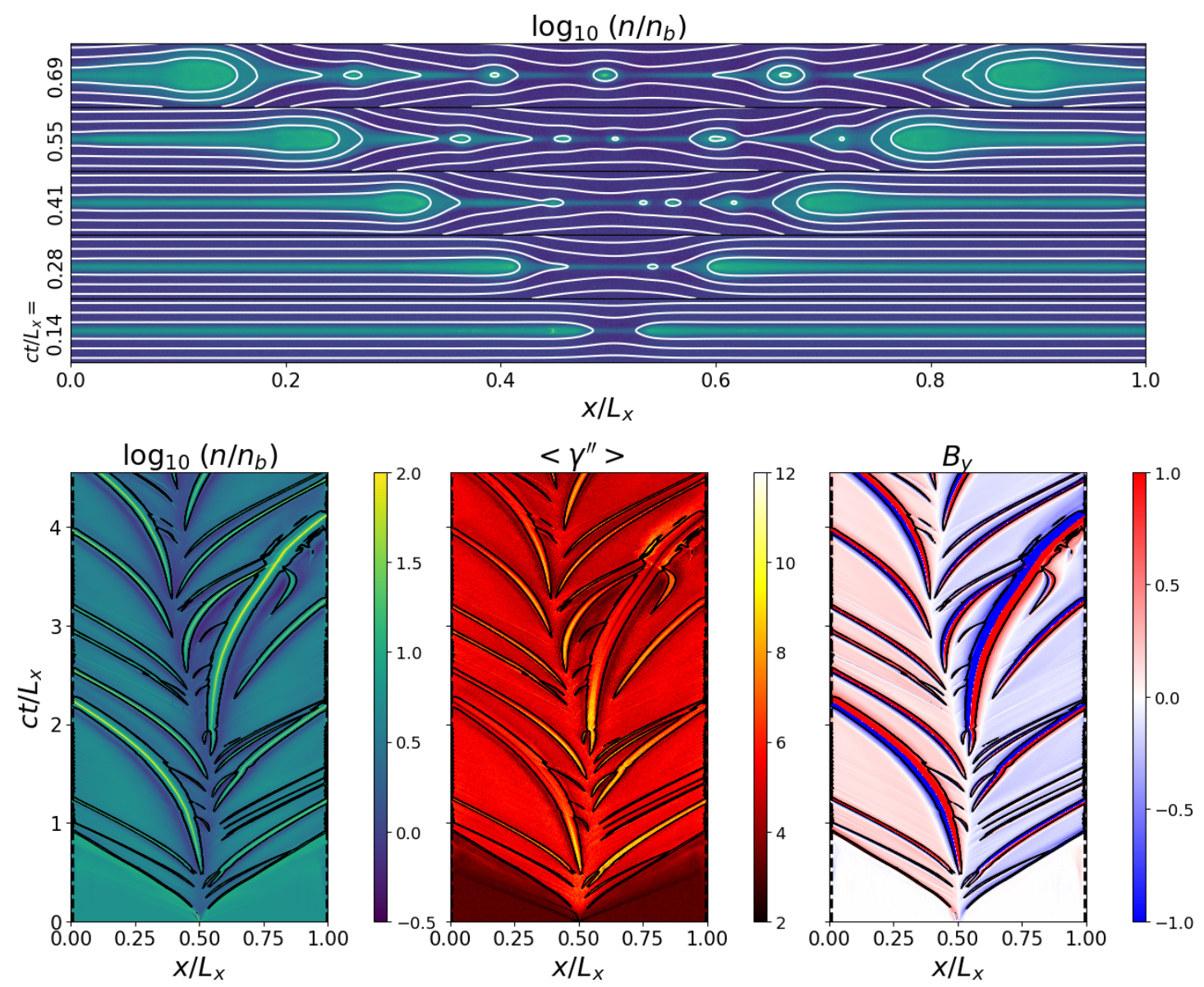

Figure 1: Top: 2D density map snapshots from the initial evolution of the reconnection layer. Bottom left: spacetime diagram of the reconnection layer density. Bottom middle: spacetime diagram of the average particle Lorentz factor calculated in the zero-momentum frame, normalized to the temperature $\Theta$ of the initial particle distribution. Bottom right: spacetime diagram of the magnetic field $y$-component.

We are interested on the processes going on within the current layer. An efficient way of presenting the different properties of the reconnecting layer are the spacetime diagrams. We collect $1 \mathrm{D}$ profiles along $\mathrm{x}$-direction of the $2 \mathrm{D}$ properties of a thin strip of the computational domain centered at the current layer averaged over y-direction. In Figure 1 we present spacetime diagrams of such 1D profiles where in the y-coordinate represents the simulation time.

At the left bottom panel we show number density. The overdensities marked with the black contours correspond to the plasmoids generated. One can observe that differences in sizes, densities and outflowing velocities appear between the plasmoids. Large plasmoids require longer times to be accelerated while small ones are rapidly accelerated up to close to Alfvén velocity. In middle panel we show the spacetime diagram of average particle Lorentz factor $\left\langle\gamma^{\prime \prime}\right\rangle$ measured 
in Landau frame, i.e. zero-momentum frame (see Werner et al., 2018). In such frame the bulk flow kinetic energy is not taken into account, then only the internal energy is presented in the diagram. We see that small plasmoids gather high energy particles without cooling down during their whole xt-trajectories. On the other hand, large plasmoids during their growth host high particle internal energy. As soon as they abandon the X-point, they start to radiate away with a greater cooling placed at their centers. The right most diagram indicates that every plasmoids enclose high magnetic field with sharp gradients within their cores. This magnetic field loops get denser as the plasmoids number density raise due to the lost of thermal pressure through cooling.

In Figure 2 it is presented the lightcurves observed at both edges of the current layer with same y-coordinate as in the spacetime diagram placed in the middle. We show two lightcurves for each observer, a lower energy band (cyan) and a higher energy band (orange). The xt-diagram presents synchrotron emission power normalized to a constant $E_{\mathrm{syn}, 0}=c\left(\sigma_{T} / 4 \pi\right) n_{\mathrm{b}} \Theta^{2} \sigma_{0}^{2} \mathrm{~d} x \mathrm{~d} y \mathrm{~d} t$. We see that the major contribution of the emission comes from plasmoids, where small ones radiate mainly homogeneously by their xt-surface and large plasmoids have differentiated emission zones in which the cores show the highest synchrotron emission power. Such great emission is observed in lightcurves which show that despite the relativistic beaming of the emission coming from small sized plasmoids, their radiation signatures are less prominent than emission coming from large sized plasmoids. Moreover, lightcurves profiles display conspicuous signatures where sharp flares at both energy bands are measured. The black dashed lines in the xt-diagram show the emission path, indicating that the sharp emission flares originate at tail-on mergers between a small/fast plasmoids and a large/slow target.

Figure 3 shows a tracked particle properties. This particle is representative of the particle ensemble that form small sized plasmoids. In the spacetime diagram the xt-path of the particle is shown with a color gradient indicating its Lorentz factor measured in the simulation frame, $\gamma$. The particle gets rapidly accelerated while staying in the X-point at y-position $y=0 \rho_{0}$, where the reconnecting layer is, as shown in the first panel of Fig. 3. Its z velocity component, $u_{z}$, increase continuously because of a systematic positive parallel electric field (measured in the plasmoid frame), $E_{\|}^{\prime}$. During this stage $B_{x}$ decrease and hence $B_{\perp}^{\prime}$ does as well, indicating low energy lost by synchrotron emission. Once the plasmoid is fully formed at outflowing, the particle trapped oscillates inside. The particle continue a weak acceleration until $c t / L_{x} \simeq 1.8$, as it is shown in the Lorentz factor measured at the plasmoid frame, $\gamma^{\prime}$, of the second panel. Although $E_{z}$ oscillates with increasing amplitude, $E_{\|}^{\prime}$ indicates a negligible net acceleration, while $B_{\perp}^{\prime}$ is positive along the path, which cause the particle to loose its kinetic energy by synchrotron emission.

Close to the computational boundary, the host plasmoid reaches a larger and slower plasmoid, interacting during $c t / L_{x} \simeq[2.10-2.15]$ and producing a sharp peak of the lightcurves measured at the right edge of the current layer. During the merge, the particle begins to gyrate attached to the edge of the target plasmoid. At this last phase, the particle encounters a continuously high amplitude $B_{x}$ and $B_{y}$ which is the source of a great centrifugal acceleration and the consequent synchrotron emission. 


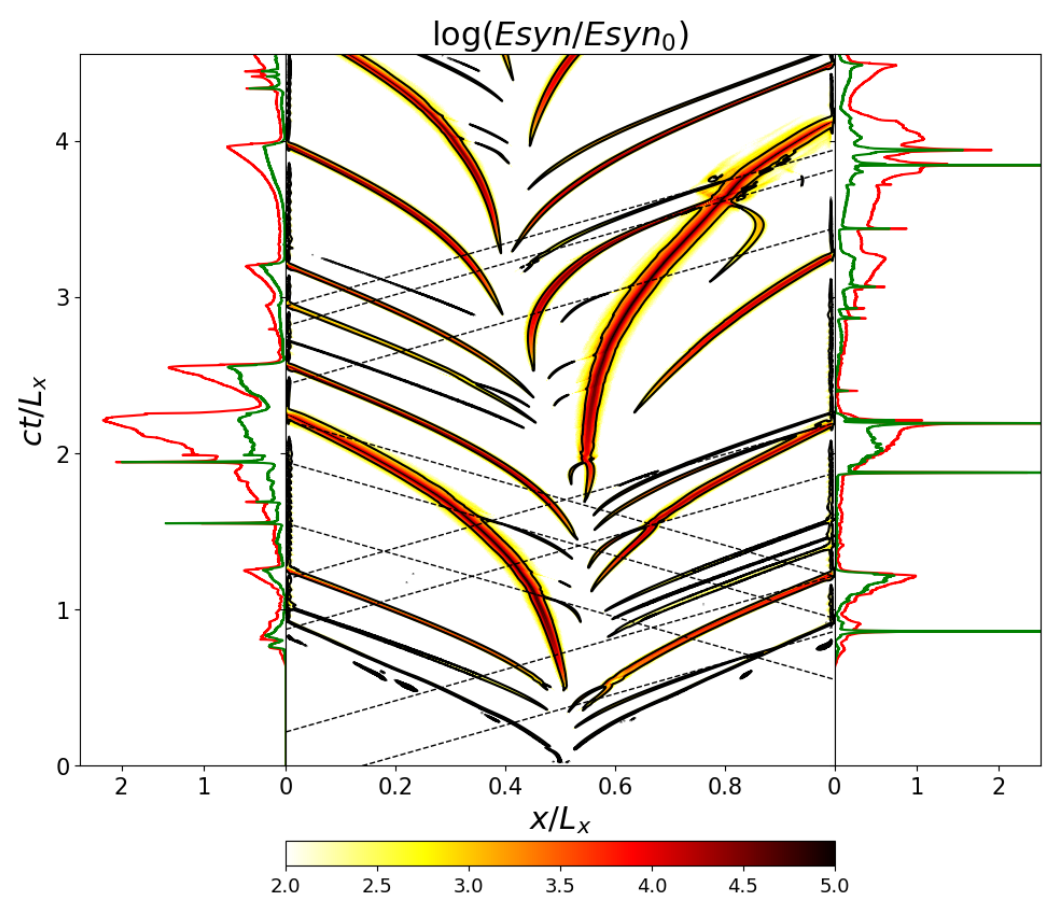

Figure 2: Middle: Spacetime diagram of the total synchrotron power. Left and right: lightcurves measured by an observer placed at the left and right, respectively, of the reconnecting layer for two frequency bands (red and green for lower and higher frequency, respectively).

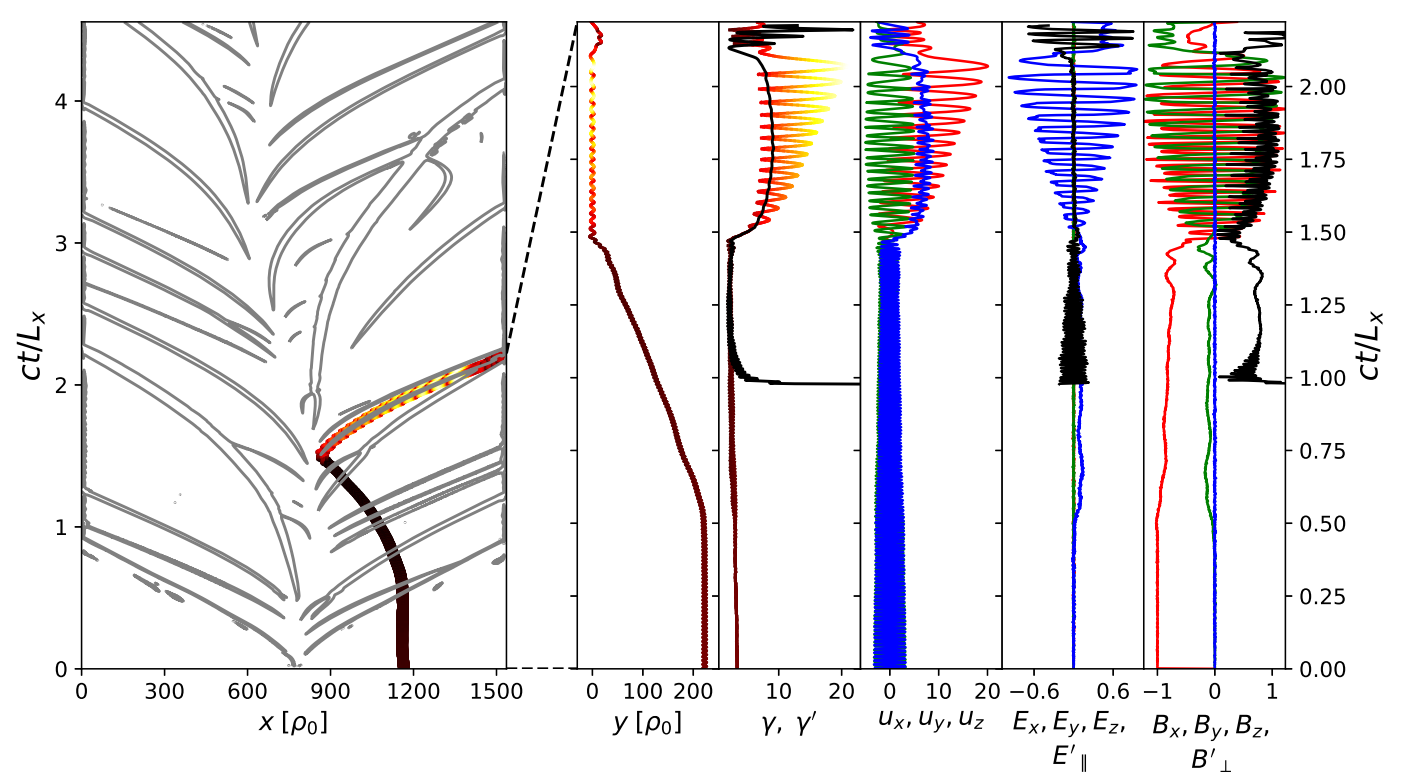

Figure 3: Acceleration history of a selected energetic particle. From left to right: x-position, y-position, Lorentz factor (normalized to $\Theta$ ), velocity vector, local electric field, and local magnetic field (x, y and $\mathrm{z}$ components are shown in red, green and blue, respectively). Parameters measured in the frame of the plasmoid to which the particle is attached, are denoted by a prime and showed in black $\left(\gamma^{\prime}, E_{\|}^{\prime}\right.$ and $\left.B_{\perp}^{\prime}\right)$. Grey contours in the first panel indicate plasma overdensities corresponding to plasmoids edges. 


\section{Conclusions}

Our results are consistent with the basic picture of steady-state relativistic plasmoid reconnection established in Sironi et al. (2016), in which the plasmoid size determines its acceleration rate, and hence large-sized plasmoids are slow, and small-sized plasmoids get rapidly accelerated. We find that under efficient radiative cooling the density of plasmoid cores is increased and the reduced gas pressure is replaced by amplified magnetic fields. Most of the synchrotron emission originates within the plasmoids cores due to such intense magnetic fields. We find that it is not possible to contain the synchrotron emission of plasmoids within the simulation domain, because they are advected out of the boundaries before they radiate their energy content. We calculate detailed synchrotron emission signatures of the plasmoid reconnection, showing that sharp emission peaks can be identified as originating from tail-on mergers between a small/fast plasmoid and a large/slow target.

Acknowledgments These results are based on numerical simulations performed at the supercomputer Prometheus located at Cyfronet AGH, Poland (PLGrid grant rec jose2 018 ; PI: K. Nalewajko). This work was supported by the Polish National Science Centre grant 2015/18/E/ST9/00580.

\section{References}

Aharonian, F., Akhperjanian, A. G., Bazer-Bachi, A. R., et al. 2007, ApJ, 664, L71

Ahnen, M. L., Ansoldi, S., Antonelli, L. A., et al. 2016, A\&A, 593, A91

Begelman, M. C., Fabian, A. C., \& Rees, M. J. 2008, MNRAS, 384, L19

Cerutti, B., Werner, G. R., Uzdensky, D. A., et al. 2013, ApJ, 770, 147

Christie, I. M., Petropoulou, M., Sironi, L., et al. 2019, MNRAS, 482, 65

Giannios, D., Uzdensky, D. A., \& Begelman, M. C. 2009, MNRAS, 395, L29

Hakobyan, H., Philippov, A., \& Spitkovsky, A. 2019, ApJ, 877, 53

Kirk, J. G., \& Skjæraasen, O. 2003, ApJ, 591, 366

Nalewajko, K., Giannios, D., Begelman, M. C., et al. 2011, MNRAS, 413, 333

Nalewajko, K. 2018, MNRAS, 481, 4342

Petropoulou, M., Giannios, D., \& Sironi, L. 2016, MNRAS, 462, 3325

Sironi, L., \& Spitkovsky, A. 2014, ApJL, 783, L21

Sironi, L., Giannios, D., \& Petropoulou, M. 2016, MNRAS, 462, 48

Schoeffler, K. M., Grismayer, T., Uzdensky, D., et al. 2019, ApJ, 870, 49

Werner, G. R., Uzdensky, D. A., Begelman, M. C., et al. 2018, MNRAS, 473, 4840 